\title{
Whitham method for the Benjamin-Ono-Burgers equation and dispersive shocks
}

\author{
Y. Matsuno, ${ }^{1, *}$ V. S. Shchesnovich, ${ }^{2, \dagger}$ A. M. Kamchatnov, ${ }^{3, \dagger}$ and R. A. Kraenkel ${ }^{4, \S}$ \\ ${ }^{1}$ Division of Applied Mathematical Science, Graduate School of Science and Engineering, Yamaguchi University, Ube 755-8611, Japan \\ ${ }^{2}$ Instituto de Física, Universidade Federal de Alagoas, Maceió Alagoas 57072-970, Brazil \\ ${ }^{3}$ Institute of Spectroscopy, Russian Academy of Sciences, Troitsk 142190, Moscow Region, Russia \\ ${ }^{4}$ Instituto de Fisica Teórica, Universidade Estadual Paulista, Rua Pamplona 145, 01405-900 São Paulo, Brazil
}

(Received 18 October 2006; published 19 January 2007)

The Whitham modulation equations for the parameters of a periodic solution are derived using the generalized Lagrangian approach for the case of the damped Benjamin-Ono equation. The structure of the dispersive shock is considered in this method.

DOI: 10.1103/PhysRevE.75.016307

PACS number(s): 47.20.-k, 47.35.Fg, 47.35.Jk, 02.90.+p

\section{INTRODUCTION}

As is known, a dispersive shock is an oscillatory structure generated in a wave system after wave breaking of an intense pulse under conditions where the dispersive effects are much greater than the dissipative ones. In this sense, such shocks are the dispersive counterparts of the usual viscous shocks well known in the dynamics of compressive viscous fluids. In surface water wave physics, dispersive shocks are known as tidal bores in rivers [1]. Besides this classical observation, dispersive shocks have also been found in some other physical systems including plasmas [2] and Bose-Einstein condensates $[3,4]$.

In typical situations, a dispersive shock can be represented as a modulated nonlinear wave whose parameters change little in one wavelength and one period; hence the Whitham modulation theory [5,6] (see also [7]) can be applied to its study. If one neglects dissipation, then a dispersive shock is a nonstationary structure expanding with time, so that at one edge it can be represented as a soliton train and at the other edge as a linear wave propagating with some group velocity into the unperturbed region. A corresponding Whitham theory of such shocks for systems described by the Korteweg-de Vries (KdV) equation was developed by Gurevich and Pitaevskii in [8] and later it was extended to other equations such as the Kaup-Boussinesq system $[9,10]$, the Benjamin-Ono (BO) equation [11-13], and the nonlinear Schrödinger equation [14]. This approach has found applications to water wave physics [15] and the dynamics of BoseEinstein condensates $[4,16]$.

The Whitham method describes the long-time evolution of the dispersive shock when many waves (crests) are generated. However, when the long-time evolution is considered, small dissipation effects can become of crucial importance. In particular, they can stop self-similar expansion of the shock so that it tends to some stationary wave structure which propagates as a whole with constant velocity. Correspondingly, the Whitham equations should be modified to

\footnotetext{
*Electronic address: matsuno@yamaguchi-u.ac.jp

${ }^{\dagger}$ Electronic address: valery@loqnl.ufal.br

*Electronic address: kamch@isan.troitsk.ru

${ }^{\S}$ Electronic address: kraenkel@ift.unesp.br
}

include the dissipation effects. This was done for the first time for the KdV-Burgers equation in $[17,18]$ by a direct method which did not apply the inverse scattering transform method to the $\mathrm{KdV}$ equation. A more general approach based on the complete integrability of unperturbed wave equations was developed in [19] and applied to the theory of bores described by the Kaup-Boussinesq-Burgers equation [20] and the $\mathrm{KdV}$ equation with Chezy friction and a bottom with a slope [21].

The method of Ref. [19] can be applied in principle to any wave equation that is completely integrable in the framework of the Ablowitz-Kaup-Newell-Segur method [22] and any perturbation depending on the wave variables and their space derivatives. However, the important $\mathrm{BO}$ equation describing internal waves in stratified deep water includes a nonlocal dispersion term and therefore it cannot be considered by the method of [19]. Although the Whitham theory for the BO equation was discussed in $[11-13,23]$, its generalization to take into account small dispersion effects has not been developed yet. The aim of this paper is to develop the Whitham theory for the Benjamin-Ono-Burgers (BOB) equation

$$
u_{t}+u u_{x}+H u_{x x}=\varepsilon u_{x x},
$$

where

$$
H u(x)=\frac{1}{\pi} \mathrm{P} \int \frac{u(y)}{y-x} d y
$$

is the Hilbert transform and the term in the right-hand side of (1) describes small friction with the viscosity parameter $\varepsilon$. In the next section we shall derive the Whitham equations which govern the slow evolution of a nonlinear periodic wave due to its modulation and small friction and in Sec. III we shall apply this theory to a stationary bore (dispersive shock).

\section{WHITHAM THEORY FOR THE BENJAMIN-ONO- BURGERS EQUATION}

The unperturbed $\mathrm{BO}$ equation has the periodic solution

$$
u(x, t)=\frac{4 k^{2}}{\sqrt{A^{2}+4 k^{2}}-A \cos \theta}+\beta, \quad \theta=k x-\omega t,
$$

which depends on three constant parameters-the wave number $k$, the amplitude of oscillations, $A=\left(u_{\max }-u_{\min }\right) / 2$, 
and $\beta$. This solution describes a nonlinear wave propagating with constant velocity

$$
V=\frac{\omega}{k}=\frac{1}{2} \sqrt{A^{2}+4 k^{2}}+\beta .
$$

In a modulated wave these three parameters become slow functions of the space and time coordinates and their evolution is governed by the Whitham equations, which were obtained in [23] for general multiphase solutions of the unperturbed BO equation by a method based on the complete integrability of the BO equation, and in $[11,12]$ for the simplest one-phase solution (3) by a direct Whitham method based on use of the Hamilton principle

$$
\delta \int d t \int d x L\left(\phi, \phi_{x}, \phi_{t}\right)=0
$$

with the Lagrangian

$$
L=\frac{1}{2} \phi_{t} \phi_{x}+\frac{1}{6} \phi_{x}^{3}+\frac{1}{2} \phi_{x} H \phi_{x x}, \quad u=\phi_{x} .
$$

In this method the periodic solution is represented in the form

$$
\phi=\psi+\Phi(\theta),
$$

where

$$
\psi=\beta x-\gamma t, \quad \theta=k x-\omega t,
$$

so that

$$
\beta=\psi_{x}, \quad \gamma=-\psi_{t}, \quad k=\theta_{x}, \quad \omega=-\theta_{t} ;
$$

hence $u=u\left(\theta, \theta_{x}, \theta_{t}, \psi_{x}\right), L=L\left(\theta, \theta_{x}, \theta_{t}, \psi_{x}, \psi_{t}\right)$, and the averaging is taken over fast oscillations according to the rule

$$
\bar{L}=\frac{1}{2 \pi} \int_{0}^{2 \pi} L d \theta,
$$

leading to an averaged Lagrangian that depends on the derivatives $\theta_{x}, \theta_{t}, \psi_{x}, \psi_{t}$. The Euler-Lagrange equations for the corresponding averaged Hamilton principle

$$
\delta \int d t \int d x \bar{L}\left(\theta_{x}, \theta_{t}, \psi_{x}, \psi_{t}\right)=0
$$

yield, taking account of (9), the Whitham equations in the form

$$
\frac{\partial}{\partial t} \frac{\partial \bar{L}}{\partial \gamma}-\frac{\partial}{\partial x} \frac{\partial \bar{L}}{\partial \beta}=0, \quad \frac{\partial}{\partial t} \frac{\partial \bar{L}}{\partial \omega}-\frac{\partial}{\partial x} \frac{\partial \bar{L}}{\partial k}=0,
$$

which should be complemented by the consistency conditions

$$
\beta_{x}+\gamma_{t}=0, \quad k_{t}+\omega_{x}=0 .
$$

After calculation of the integral (10) they reduce to a system of equations for the parameters $\beta, k, V=\omega / k$ (see [11,12]).

Now our task is to generalize this procedure to the perturbed BO equation (1). Instead of using the Lagrangian formulation with an additional field (see, for instance, Ref. [24]) we prefer to introduce another approach which does not require introduction of new auxiliary fields. We propose to use directly the Hamilton principle in its infinitesimal form by noticing that Eq. (1) can be written symbolically as

$$
\int d t \int d x\left\{\delta L+\varepsilon \phi_{x x x} \delta \phi\right\}=0,
$$

where $\delta L$ is a variation of the Lagrangian (6). Now we can transform (14) in the following way. First, we integrate the "dissipative" term by parts and use $u=\phi_{x}$ :

$$
\int d t \int d x\left\{\delta L-\varepsilon u_{x} \delta u\right\}=0 .
$$

Second, we average the dissipative term as follows:

$$
\begin{aligned}
\int d x \overline{\left(u_{x} \delta u\right)}= & \int d x \overline{\left[u_{x}\left(u_{\theta} \delta \theta+u_{\theta_{x}} \delta \theta_{x}+u_{\theta_{t}} \delta \theta_{t}+u_{\psi_{x}} \delta \psi_{x}\right)\right]} \\
= & \int d x\left\{\overline{\left[u_{x} u_{\theta}-\frac{\partial}{\partial x}\left(u_{x} u_{\theta_{x}}\right)-\frac{\partial}{\partial t}\left(u_{x} u_{\theta_{t}}\right)\right]} \delta \theta\right. \\
& \left.-\frac{\partial}{\partial x} \overline{\left(u_{x} u_{\psi_{x}}\right)} \delta \psi\right\} .
\end{aligned}
$$

In the Whitham approximation with fast $\theta$ variable we have $\partial / \partial x \cong \theta_{x} \partial / \partial \theta, \partial / \partial t \cong \theta_{t} \partial / \partial \theta$, where within the averaging interval the parameters $\theta_{x}$ and $\theta_{t}$ can be considered constant, the terms with $\theta$-derivatives become equal to zero after averaging, and, hence, we arrive at the expression

$$
\int d t \int d x \overline{\left(u_{x} \delta u\right)}=\int d t \int d x \overline{\left(u_{x} u_{\theta}\right)} \delta \theta=\int d t \int d x \overline{k\left(u_{\theta}^{2}\right)} \delta \theta .
$$

Transformation of the term with the Lagrangian in (15) can be performed in a similar way and as a result we obtain the Whitham equations in the form

$$
\frac{\partial}{\partial t} \frac{\partial \bar{L}}{\partial \gamma}-\frac{\partial}{\partial x} \frac{\partial \bar{L}}{\partial \beta}=0, \quad \frac{\partial}{\partial t} \frac{\partial \bar{L}}{\partial \omega}-\frac{\partial}{\partial x} \frac{\partial \bar{L}}{\partial k}=\varepsilon k \overline{\left(u_{\theta}^{2}\right)},
$$

which generalize Eqs. (12) to the BOB equation (1).

A simple calculation of the averaged values gives $[11,12]$

$$
\begin{gathered}
\bar{L}=\frac{1}{3} k^{3}-k\left(\frac{\omega^{2}}{k^{2}}-\frac{\beta \omega}{k}+\gamma\right)+\frac{1}{6} \beta^{3}-\frac{1}{2} \beta \gamma, \\
\varepsilon k \overline{\left(u_{\theta}^{2}\right)}=2 \varepsilon(V-\beta)\left[(V-\beta)^{2}-k^{2}\right] .
\end{gathered}
$$

Their substitution into (17) and use of (13) permit one to express $\gamma$ as $\gamma=\beta^{2} / 2$ and to transform the equations for the other parameters to the forms

$$
\begin{gathered}
\beta_{t}+\beta \beta_{x}=0, \\
k_{t}+(V k)_{x}=0, \\
V_{t}+V V_{x}+k k_{x}=-\varepsilon(V-\beta)\left[(V-\beta)^{2}-k^{2}\right] .
\end{gathered}
$$

These are the Whitham equations for the physical parameters $\beta, k, V$. We note that these equations can also be derived by 
means of averaging of modified conservation laws. For the BO equation without dissipation, such a derivation of the Whitham equations was given in [12].

Although Eqs. (20) are simple enough for further investigations, they can be transformed to the theoretically more attractive diagonal form by introduction of Riemann invariants $a, b, c$ according to the definitions

$$
\beta=2 c, \quad k=b-a, \quad V=b+a \quad(c<a<b),
$$

so that we get the system

$$
\begin{gathered}
a_{t}+2 a a_{x}=-2 \varepsilon(a-c)(b-c)(a+b-2 c), \\
b_{t}+2 b b_{x}=-2 \varepsilon(a-c)(b-c)(a+b-2 c), \\
c_{t}+2 c c_{x}=0 .
\end{gathered}
$$

In terms of Riemann invariants the periodic solution (3) takes the form

$$
u(x, t)=\frac{2(b-a)^{2}}{a+b-2 c-2 \sqrt{(a-c)(b-c)} \cos \theta}+2 c,
$$

where

$$
\theta=(b-a) x-\left(b^{2}-a^{2}\right) t .
$$

The parameters $a, b, c$ must satisfy the condition $2 c<a+b$ to keep the solution nonsingular. The amplitude of oscillations is expressed as

$$
A=4 \sqrt{(a-c)(b-c)} .
$$

When for some concrete problem the solution of Eqs. (22) is found and the functions $a=a(x, t), b=b(x, t), c=c(x, t)$ are known, their substitution into (23) yields the modulated nonlinear wave for the problem under consideration. The mean value of $u$ in this oscillatory region is equal to

$$
\bar{u}=2 k+\beta=2(b+c-a) .
$$

In the next section we shall consider an example of such a problem.

\section{DISPERSIVE SHOCK (BORE) IN INTERNAL WAVES IN A DEEP FLUID}

As was mentioned in the Introduction, the dispersive shock is an oscillatory region joining two regions with different values of the wave amplitude $u_{ \pm}$which arise after wave breaking. In the simplest case of the GurevichPitaevskii problem one can consider $u_{ \pm}$as two constants: $u \rightarrow u_{ \pm}$as $x \rightarrow \pm \infty$, respectively, and without loss of generality we can take $u_{+}=0$ and denote $u_{-}=u_{0}=$ const. Thus, we have to find the solution of the Whitham equations that corresponds to a modulated nonlinear wave satisfying the boundary conditions

$$
u \rightarrow \begin{cases}u_{0}, & x \rightarrow-\infty, \\ 0, & x \rightarrow+\infty\end{cases}
$$

Naturally, the initial profile of the wave $u=u(x, 0)$ must also satisfy this condition, and an easy calculation shows that

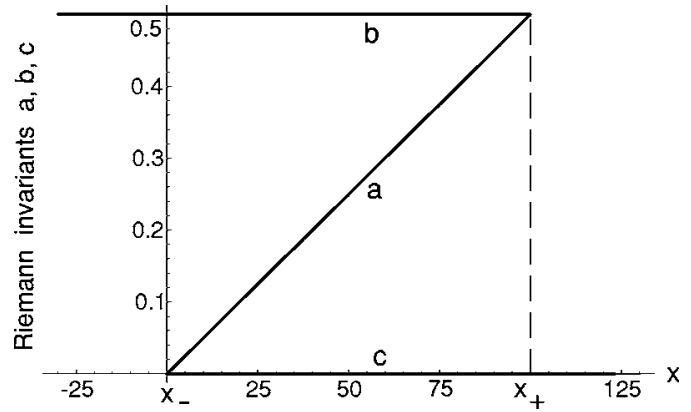

FIG. 1. Plots of the Riemann invariants at $t=100$ as functions of $x$ in the case of decay of the steplike initial distribution without friction.

after wave breaking the waves are generated with wavelength $L \sim 1 / u_{0}$.

Now, we can distinguish two typical stages of evolution of the wave: (1) the initial stage for $t \ll 1 /\left(\varepsilon u_{0}^{2}\right)$, when we have $\left|u_{t}\right| \gg \varepsilon\left|u_{x x}\right|$, so that one can neglect a viscous term in (1); and (2) the asymptotic stage of large time $t \gg 1 /\left(\varepsilon u_{0}^{2}\right)$, when the solution tends to the stationary solution determined by interplay of the dispersion and dissipation effects. Examples of the first stage have already been studied in $[12,13]$. The simplest case of a steplike initial condition has been discussed in [11], and we shall reproduce some results here in Riemann invariant form and with additional remarks for convenience of future comparison with the second stage of asymptotically large time.

Thus, we suppose that at the initial moment $t=0$ the region of transition from $u=u_{0}$ to $u=0$ is very narrow (i.e., much less than $L \sim 1 / u_{0}$ ) so that the initial profile can be approximated by a steplike function,

$$
u(x, 0)= \begin{cases}u_{0}, & x<0, \\ 0, & x>0 .\end{cases}
$$

As was noticed above, at the initial stage we can neglect the dissipation effects, so that the dispersive shock arising is governed by the equations

$$
a_{t}+2 a a_{x}=0, \quad b_{t}+2 b b_{x}=0, \quad c_{t}+2 c c_{x}=0 .
$$

After averaging over the wavelength the initial-value problem for the Riemann invariants does not contain any parameters with dimension of length. Hence, the Riemann invariants can depend on the self-similar variable $\zeta=x / t$ only so that the solution of Eqs. (29) has the form (see Fig. 1)

$$
\begin{gathered}
a=x / 2 t, \quad 0<x<x_{+}, \\
b=u_{0} / 2, \quad x<x_{+}, \\
c=0, \quad x>x_{-}=0,
\end{gathered}
$$

where

$$
x_{+}=u_{0} t .
$$

Substitution of this solution into (23) gives the expression for $u$ in the oscillatory region, 


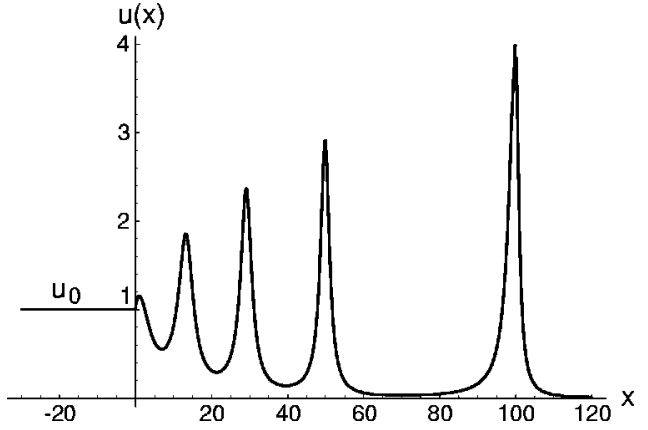

FIG. 2. Profile of the bore at $t=100$ corresponding to selfsimilar decay of the steplike initial distribution without friction.

$$
u(x, t)=\frac{\left(u_{0} t-x\right)^{2}}{t\left(u_{0} t+x-2 \sqrt{u_{0} t x} \cos \theta\right)},
$$

where

$$
\theta=\left[2\left(u_{0} t-x\right) x-\left(u_{0}^{2} t^{2}-x^{2}\right)\right] /(4 t) .
$$

The corresponding plot of the dispersive shock profile at fixed $t$ is shown in Fig. 2. It agrees with the direct numerical solution of the BO equation with a steplike initial condition (28) which was presented in Ref. [25]. At the leading front we can see a soliton with amplitude

$$
u_{s}=4 V=4 u_{0},
$$

which moves to the right with velocity $V=u_{0}$. The trailing edge is located at $x=0$ and corresponds to a linear wave with vanishing amplitude and zero value of the group velocity. Indeed, linearization of the $\mathrm{BO}$ equation with respect to the small amplitude $A$ in $u \cong U_{0}+A \cos (k x-\omega t)$ leads to the dispersion relation

$$
\omega=u_{0} k-k^{2} .
$$

According to (21) we have $k=b=u_{0} / 2$ at $x=0$ and hence

$$
\left.\frac{d \omega}{d k}\right|_{k=u_{0} / 2}=0 \quad \text { at } x=0 .
$$

The number of waves in the oscillatory region is equal to

$$
N=\frac{1}{2 \pi} \int_{0}^{u_{0} t} k d x=\frac{u_{0}^{2} t}{8 \pi} .
$$

The self-similar expansion of the oscillatory region holds as long as the viscosity effects can be neglected. However, these effects come into play at $t \sim 1 /\left(\varepsilon u_{0}^{2}\right)$ and at $t \rightarrow \infty$ the shock profile tends to the stationary structure propagating with constant velocity. To find this structure, we look for the stationary solution of the Whitham equations (22) so that the Riemann invariants are functions of $\xi=x-V t$ only, where $V=a+b=$ const. We assume that $\xi=0$ corresponds to the leading soliton front of the shock, where $k=b-a=0$ and $\bar{u}=2(b+c-a)=0$, which give at once that $c=0$ and $a=b=V / 2$ at $\xi=0$. Then the last equation (22) gives $c=0$ identically and the remaining equations (22) reduce to a single equation

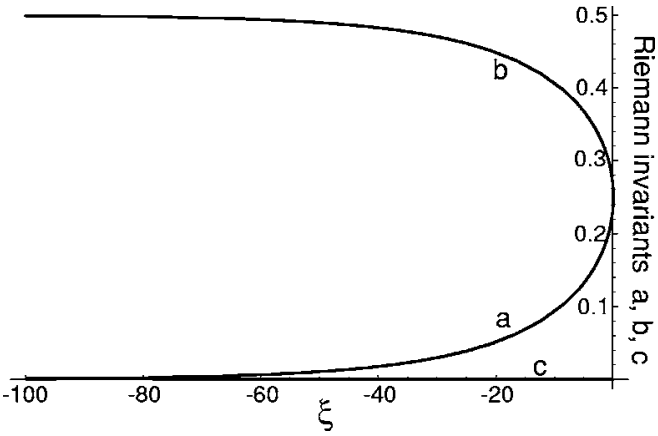

FIG. 3. Plots of the Riemann invariants as functions of $\xi$ in the case of the stationary bore solution with friction $(\varepsilon=0.05)$.

$$
(2 b-V) b_{\xi}=-2 \varepsilon V b(V-b),
$$

which should be solved with the initial condition

$$
\left.b\right|_{\xi=0}=\frac{V}{2} .
$$

An elementary calculation taking account of the inequality $b>a=V-b$, i.e., $b \geq V / 2$, gives at once $b=(V / 2)[1+\sqrt{1-\exp (2 \varepsilon V \xi)}]$. Finally, at $\xi \rightarrow-\infty$ we must have $a=0, b=V, \bar{u}=u_{0}=2 b=2 V$, that is, $V=u_{0} / 2$, and we arrive at the solution

$$
\begin{gathered}
a=\frac{u_{0}}{4}[1-\sqrt{1-\exp (2 \varepsilon V \xi)}], \\
b=\frac{u_{0}}{4}[1+\sqrt{1-\exp (2 \varepsilon V \xi)}], \quad c=0,
\end{gathered}
$$

where $\xi=x-\left(u_{0} / 2\right) t$. Plots of the Riemann invariants are shown in Fig. 3; they should be compared with Fig. 1. Substitution of Eq. (40) into Eq. (23) yields the profile of the shock,

$$
u(x, t)=\frac{u_{0}\left[1-\exp \left(\varepsilon u_{0} \xi\right)\right]}{1-\exp \left(\varepsilon u_{0} \xi / 2\right) \cos \theta}
$$

where

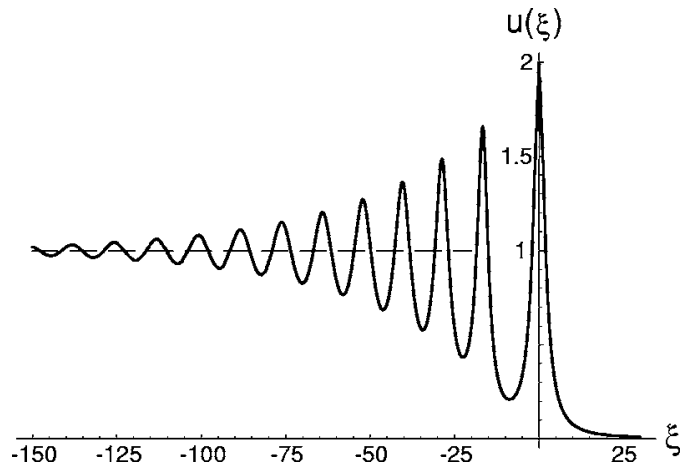

FIG. 4. Profile of the stationary bore described by the BebjaminOno-Burgers equation with friction coefficient $\varepsilon=0.05$. Dashed line corresponds to the constant value of $u$ at $x \rightarrow-\infty$. 


$$
\theta=\frac{u_{0}}{2} \sqrt{1-\exp \left(\varepsilon u_{0} \xi\right)} \xi, \quad \xi=x-\frac{u_{0}}{2} t
$$

The corresponding plot is shown in Fig. 4. Again at the leading front we can see a soliton, but now it has the amplitude

$$
u_{s}=2 u_{0}
$$

and propagates with velocity $V=u_{0} / 2$. Thus, the friction effects lead to decrease of the amplitude and velocity of the soliton compared with the nonstationary stage. However, it is important to notice that, in contrast to an isolated soliton, the profile of the bore becomes asymptotically stationary. In this stationary solution the trailing edge is located at $x=-\infty$, which corresponds to the position of the initial discontinuity in this reference frame: $x \cong-\left(u_{0} / 2\right) t$ at $t \rightarrow \infty$. Hence, even when the leading front has almost reached its stationary profile, the rear edge of the shock wave still remains a nonstationary wave packet located at the position of the initial steplike discontinuity.

\section{CONCLUSION}

In this paper, we have discussed the structure of the dispersive shock described by the BO equation taking account of small friction effects. It is shown that there exists a stationary profile so that the nonstationary oscillating structure supported by a jump of the wave amplitude at two spatial infinities tends asymptotically to this stationary profile.

The Whitham method applied to this problem is very simple in the case of the BOB equation, and the corresponding equations can be solved in elementary and explicit form.

\section{ACKNOWLEDGMENTS}

The work of V.S.S. was supported by a CNPq grant. A.M.K. thanks FAPESP for support of his stay at IFTUNESP, Brazil. V.S.S. thanks the IFT-UNESP for warm hospitality and financial support during his visit.
[1] B. Benjamin and M. J. Lighthill, Proc. R. Soc. London, Ser. A 224, 448460 (1954).

[2] M. Khan, S. Ghosh, S. Sarkar, and M. R. Gupta, Phys. Scr., T 116, 5356 (2005).

[3] T. P. Simula, P. Engels, I. Coddington, V. Schweikhard, E. A. Cornell, and R. J. Ballagh, Phys. Rev. Lett. 94, 080404 (2005).

[4] M. A. Hoefer, M. J. Ablowitz, I. Coddington, E. A. Cornell, P. Engels, and V. Schweikhard, Phys. Rev. A 74, 023623 (2006).

[5] G. B. Whitham, Proc. R. Soc. London, Ser. A 283, 238 (1965).

[6] G. B. Whitham, Linear and Nonlinear Waves (WileyInterscience, New York, 1974).

[7] A. M. Kamchatnov, Nonlinear Periodic Waves and Their Modulations-An Introductory Course (World Scientific, Singapore, 2000).

[8] A. V. Gurevich and L. P. Pitaevskii, Zh. Eksp. Teor. Fiz. 65, 590 (1973) [Sov. Phys. JETP 38, 291 (1973)].

[9] G. A. El, R. H. J. Grimshaw, and M. V. Pavlov, Stud. Appl. Math. 106, 157 (2001).

[10] G. A. El, R. H. J. Grimshaw, and A. M. Kamchatnov, Stud. Appl. Math. 114, 395 (2005).

[11] Y. Matsuno, J. Phys. Soc. Jpn. 67, 1814 (1998).

[12] Y. Matsuno, Phys. Rev. E 58, 7934 (1998).
[13] M. C. Jorge, A. A. Minzoni, and N. F. Smyth, Physica D 132, 1 (1999).

[14] A. M. Kamchatnov, R. A. Kraenkel, and B. A. Umarov, Phys. Rev. E 66, 036609 (2002).

[15] J. P. Apel, J. Phys. Oceanogr. 33, 2247 (2003).

[16] A. M. Kamchatnov, A. Gammal, and R. A. Kraenkel, Phys. Rev. A 69, 063605 (2004).

[17] A. V. Gurevich and L. P. Pitaevskii, Sov. Phys. JETP 66, 490 (1987).

[18] V. V. Avilov, I. M. Krichever, and S. P. Novikov, Sov. Phys. Dokl. 32, 564 (1987).

[19] A. M. Kamchatnov, Physica D 188, 247261 (2004).

[20] G. A. El, R. H. J. Grimshaw, and A. M. Kamchatnov, Chaos 15, 037102 (2005).

[21] G. A. El, R. H.J. Grimshaw, and A. M. Kamchatnov (unpublished).

[22] M. J. Ablowitz, D. J. Kaup, A. C. Newell, and H. Segur, Stud. Appl. Math. 53, 249 (1974).

[23] S. Yu. Dobrokhotov, and I. M. Krichever, Math. Notes 49, 583 (1991).

[24] D. J. Kaup and B. A. Malomed, Physica D 87, 155 (1995).

[25] D. R. Christie, J. Atmos. Sci. 46, 1462 (1989). 